\title{
RELAÇÃO ENTRE O CONSUMO DE EMBUTIDOS DE CARNE E O DESENVOLVIMENTO DE CÂNCER NO TRATO DIGESTÓRIO
}

\author{
RELATIONSHIP BETWEEN THE CONSUMPTION OF FILLED MEAT AND THE \\ CAUSATION OF CANCER IN THE DIGESTORY TRACT
}

\author{
Wanderson Cosme da Silva ${ }^{1}$ \\ Thiago Teixeira de Oliveira Gomes ${ }^{2}$ \\ Edmundo Moreira ${ }^{3}$
}

RESUMO: O presente estudo trata-se de uma revisão de literatura sobre o desenvolvimento de patologias oncológicas do trato digestório a partir de altos consumos de embutidos de carne de origem bovina ou suína. Trata-se, portanto de uma revisão integrativa sobre esse tema. A revisão da literatura indica que compostos químicos decorrentes do processamento das carnes seja por defumação, cura, salga ou por adição de conservantes químicos podem danificar as células do cólon e do reto, que absorvem compostos químicos (nitritos, nitratos, aminas heterocíclicas e aminas policíclicas) que acabam provocando o desenvolvimento de pólipos. Esses pólipos podem ser inofensivos, porém com passagem constante dos aditivos nitrito e nitrato pelo trato gastrointestinal, ou seja, consumo excessivo dos embutidos, eles aumentam o risco desses pólipos apresentarem malignidade.

Palavras-chave: Carne vermelha Câncer colorretal. Nitritos. Nitratos.

ABSTRACT: The present study is a literature review on the development of oncological pathologies of the gastrointestinal tract or in the colon from high consumption of sausages from beef or pork. It is, therefore, an integrative review on this topic. The literature review indicates that chemical compounds resulting from meat processing, whether by smoking, curing, salting or by adding chemical preservatives, can damage colon and rectal cells, which absorb chemical compounds (nitrites, nitrates, heterocyclic amines and polycyclic amines) that end up causing the development of polyps. These polyps can be harmless, but with constant passage of nitrite and nitrate additives through the gastrointestinal tract, that is, excessive consumption of sausages, they increase the risk of these polyps presenting malignancy.

Keywords: Red meat. Processed meat. Colorectal câncer. Nitrites. Nitrates.

\footnotetext{
${ }^{I}$ Professor orientador, Mestre em Ciências da Saúde. Universidade São Judas Tadeu. Unidade MOOCA.

${ }^{2}$ Graduando de Biomedicina. Universidade São Judas Tadeu. Unidade MOOCA.

${ }^{3}$ Graduando de Biomedicina. Universidade São Judas Tadeu. Unidade MOOCA.
} 


\section{INTRODUÇÃO}

O câncer colorretal é uma neoplasia que fere os segmentos do intestino. Ele possui sua identificação pela Classificação Internacional de Doença, com sua subdivisão em neoplasias de cólon, da junção retossigmoide, do reto e do ânus. (MENEZES et al, 2016).

O câncer colorretal é considerado um grave problema na saúde pública, ele é identificado como o terceiro tipo de câncer mais presente e comum no mundo sendo mais presente em países bem desenvolvidos, e com menores acometimentos em regiões como na África e América Latina. Nas regiões Sul e Sudeste do Brasil este tipo de câncer já é comprovado como a terceira causa de morte por câncer. (MENEZES et al, 2016).

Segundo os dados do Instituto Nacional do Câncer, a estimativa de novos casos no Brasil para cada ano do triênio de 2020-2022 é de 20.540 casos de câncer de cólon e reto em homens e 20.470 em mulheres. O câncer de cólon e reto em homens, é o terceiro mais frequente, e apresenta a seguinte incidência, por roo mil habitantes, nas regiões do Brasil: Regiões Sudeste $(28,62)$, Centro-Oeste $(15,40)$, na Região Sul $(25,11)$, enquanto nas Regiões Nordeste $(8,91)$ e Norte $(5,43)$. Para as mulheres, é o segundo mais frequente nas Regiões Sudeste (26,18/10o mil) e Sul (23,65/100 mil). Nas Regiões Centro-Oeste (15,24/10o mil), Nordeste (ıo,79/ıo mil) e Norte (6,48/ıo mil) é o terceiro mais incidente (INCA, 2020). O câncer é uma das principais causas de morte em todo o mundo, sendo responsável por quase io milhões de mortes em 2020. O câncer colorretal, em 2020, foi o terceiro mais comum em termos de novos casos de câncer - I,93 milhão de casos, e o segundo mais comum em causa de morte por câncer - 935.00o mortes (WHO, 2021).

Evidências têm se acumulado ao longo dos anos de que carnes processadas e embutidos causam câncer. Existem três produtos químicos em particular que foram associados ao câncer colorretal. Um desses produtos químicos ocorre naturalmente na carne, os demais se desenvolvem ou são adicionados como parte do processo de produção dessas carnes, são eles: Heme, um pigmento encontrado principalmente na carne vermelha; Nitratos e nitritos que são adicionados para manter a carne processada com aparência de fresca por mais tempo, eles também inibem o crescimento do Clostridium Botulinum (FRANCO, LANDGRAF, 2016); e, as aminas heterocíclicas e as aminas policíclicas que são produzidas quando a carne é cozida em altas temperaturas. Todos esses 
produtos químicos podem danificar as células do cólon e do reto. À medida que os danos se acumulam ao longo do tempo, o risco de câncer aumenta muito. (UNDERFERTH,2016).

O Instituto Americano de Pesquisa do Câncer alerta que esta patologia pode não ser proveniente apenas do consumo desses tipos de embutidos e faz recomendações para que evitem ao máximo essas carnes processadas, ou seja comer o mínimo. Uma vez que qualquer carne processada (conservada, curada ou passada por processo de defumação) apresenta o risco de desenvolvimento de câncer no sistema digestório (UNDERFERTH,2016).

\section{OBJETIVOS}

Identificar e descrever, por meio de uma revisão de literatura descritiva narrativa, os principais aspectos epidemiológicos, e a relação entre o desenvolvimento de carcinomas devido a ingestão de alimentos denominados embutidos. Os embutidos de maneira geral são carnes aromatizadas com diferentes especiarias e ervas aromáticas, introduzidas em tripas de animais (naturais) ou em tripas artificiais, e processadas para alongar sua vida útil (FOOD SAFETY BRAZIL,2019). O processamento das carnes e o uso de compostos químicos conservantes podem quando passando pelo trato intestinal ocasionar, com o tempo, neoplasias nas células presentes, gerando o câncer colorretal. O presente estudo possui como objetivo mostrar que o consumo de carnes processadas, como salsicha, linguiça, bacon e presunto, pode ser prejudicial ao organismo com o decorrer do tempo e condição em que esses alimentos são ingeridos aumentando o risco e incidência do câncer colorretal.

\section{REVISÃO DE LITERATURA}

\section{EMBUTIDOS}

A origem dos embutidos vem de técnicas de preservação das carnes para um consumo posterior. Inicialmente eram obtidos pela mistura de carnes magras e gorduras que eram introduzidas em tripas dos próprios animais, utilizando o sal para desidratar e conservar a carne e defumando para aumentar a durabilidade. Ao longo dos séculos esses processos foram aperfeiçoados com a adição de especiarias que alongaram mais sua vida útil e melhoraram o paladar (FOOD SAFETY BRAZIL,2019). 
Atualmente, as carnes processadas não são apenas os embutidos, mas utilizam os mesmos processos de preparação: defumação, cura, salga e adição de compostos químicos conservantes.

Esses produtos são convenientes, acessíveis e integrados em nossa dieta diária: presunto, salsicha, pepperoni, carne seca, rosbife, blanquet de peru, linguiças, entre outros. Infelizmente, quando essas carnes são processadas, formam-se substâncias cancerígenas. (UNDERFERTH, 2016).

\section{CÂNCER DE INTESTINO}

De acordo com as estimativas mais recentes do Global Burden of Disease Project, uma organização de pesquisa acadêmica independente, cerca de 34.000 mortes por câncer por ano em todo o mundo são atribuíveis a dietas ricas em carne processada (HARVARD, 2015).

Durante o processamento, as carnes processadas são submetidas a altas temperaturas, resultando na produção de aminas heterocíclicas e hidrocarbonetos policíclicos aromáticos com potencial carcinogênico em pessoas com predisposição genética (INCA, 202I).

Recomenda-se limitar o consumo de carne vermelha a menos de 500 gramas de carne cozida por semana (aproximadamente 700-75og do peso cru). Uma das possíveis explicações para essa associação é que as carnes vermelhas são fontes importantes de ferroheme, nutriente essencial ao corpo, mas que, em excesso, pode levar à formação de compostos $\mathrm{N}$-nitrosos e de formas alcenais citotóxicas oriundas da peroxidação lipídica (INCA, 202I).

Estudos publicados na última década têm propiciado um maior conhecimento no papel da dieta na morbimortalidade por neoplasias. Observou-se que uma alimentação rica em carne vermelha, gordura animal e alimentos enlatados e embutidos estão diretamente ligados ao câncer gástrico, de cólon e reto (BECKEL HENDGES et al, 2017).

No caso do câncer de intestino (cólon e reto), até 75\% dos casos é resultante da ação cumulativa de agentes carcinógenos (INCA, 2003). O câncer colorretal está associado a dietas ricas em proteínas e gorduras; também destaca que aditivos alimentares e métodos 
de preparação do alimento também contribuem para o desenvolvimento deste câncer (SPRINGHOUSE, 2004).

Uma análise de dados de ro estudos estimou que cada porção de 50 gramas de carne processada ingerida diariamente aumenta o risco de câncer colorretal em cerca de $18 \%$ (HARVARD, 2015).

A indução a formação tumoral dos compostos químicos nitrito e nitrato foram documentadas uma vez que esses compostos, por meio da transformação química ao se tornar o composto nitrito, leva ao aumento de lesão celular devido a produção maior de radicais livres. (GARÓFOLO et al, 2004).

A conservação de carnes curadas com nitrito de sódio é provedora do nitrito, que também pode ser formado endogenamente, além disso, o nitrito provém dos vegetais como: beterraba, cenoura, nabo e entre outros que possuem nitrato e sem esquecer dos próprios alimentos embutidos. Os nitratos ingeridos são convertidos em nitrito através da própria ação da saliva. $\mathrm{O}$ aumento do risco do câncer colorretal vem de acordo com a associação do consumo de compostos nitrosos que fazem o aumento de radicais livres, e esses reduzem a produção de muco, logo sem essa proteção da mucosa gástrica, começa a acontecer as lesões celulares. (GARÓFOLO et al, 2004). O nitrito se reduz a óxido nitroso, que, por sua vez, retarda o crescimento do Clostridium botulinum e a conseqüente produção da enterotoxina durante o armazenamento. Sua eficiência bacteriostática está relacionada com o pH do alimento, que determina a concentração da forma não dissociada do ácido nitroso, sendo mais eficiente em $\mathrm{pH}$ igual ou inferior a 5,o. $\mathrm{O}$ nitrato não apresenta nenhuma atividade inibidora contra o Clostridium botulinum, mas sua ação é manifestada após sua redução a nitrito pelas bactérias presentes na carne (RIBEIRO et al, 2004).

Métodos de preservação e preparo de carnes, que acarretam a formação de aminas heterocíclicas, além dos nitritos, também foram associados ao maior risco de cânceres do trato gastrointestinal. Por exemplo, preparar as carnes com temperaturas elevadas, produzindo um suco queimado, ou expor a carne diretamente ao fogo, como durante o preparo do churrasco, tem sido desaconselhado pela World Cancer Research Fund, por produzir componentes carcinogênicos na superfície do alimento e aumentar o risco de câncer do estômago. A presença de elevada quantidade de benzo [a] pirenos - 
hidrocarboneto cíclico aromático nos alimentos, considerado um potente carcinogênico também é correlacionada com essas formas de preparações e parece associar-se com o risco aumentado de câncer do trato gastrintestinal (GARÓFOLO et al, 2004).

São fatores de risco para o desenvolvimento de câncer colorretal: histórico familiar de câncer colorretal (CCR): idade; dieta baseada em gorduras animais; baixa ingestão de frutas, vegetais e cereais integrais; etilismo e tabagismo; obesidade e sedentarismo. $\mathrm{O}$ alto consumo de peixes, o baixo consumo de carnes vermelhas processadas e a prática de exercícios físicos são fatores protetores. Com relação à idade, o CCR é mais frequente entre idosos, principalmente após os 60 anos, embora possa surgir em qualquer idade, sendo mais agressivo quanto mais precoce for o seu surgimento e, geralmente, relacionado à transmissão hereditária. As doenças inflamatórias intestinais, em especial a retocolite ulcerativa, também se relacionam com CCR. Os pólipos adenomatosos esporádicos, responsáveis por $90 \%$ de todos os casos de câncer de cólon e reto, são condições préneoplásicas, havendo transformação do epitélio do cólon normal em pólipo adenomatoso. A progressão adenocarcionoma é lenta e dura em média dez anos, fazendo com que esse tipo de neoplasia seja o alvo ideal de programas de prevenção e rastreamento da população (MENEZES et al, 2016).

O CCR geralmente é assintomático, entretanto, devem ser valorizados os sintomas e sinais de alerta, tais como: alteração do hábito intestinal, dor abdominal, sangue oculto e alterações nas fezes. Os menos comuns são presença de muco nas fezes, dor no baixo ventre, anemia, queda do estado geral, tumor abdominal palpável, obstrução intestinal aguda, fístulas colônicas e peritonite fecal por perfuração intestinal, entretanto, fazem parte do quadro clínico da doença. É considerado um dos cânceres que mais respondem às medidas de prevenção (controle dos fatores de risco e o rastreamento precoce). A detecção e remoção dos pólipos através de exames de rastreio tornam-se um importante método de prevenção, sendo a colonoscopia o procedimento de escolha para triagem e tratamento dessas lesões (MENEZES et al, 2016).

Em pacientes com baixo risco de desenvolver CCR, o rastreio deve ser realizado com pesquisa anual de sangue oculto nas fezes, sigmoidoscopia flexível a cada cinco anos ou retossigmoidoscopia rígida a cada dois anos para indivíduos a partir dos 50 anos de 
idade. Os pacientes com alto risco de desenvolver CCR devem ser rastreados com colonoscopia a partir dos 40 anos de idade (MENEZES et al, 2016).

O consumo de carnes processadas, como salsicha, linguiça, bacon e presunto, aumenta o risco de câncer do intestino em humanos, afirma relatório da Organização Mundial da Saúde (OMS). De acordo com o documento, a carne processada é um fator de risco certo para a doença, e carnes vermelhas, de um modo geral, são fatores de risco "provável" (INCA, 2015).

A associação de embutidos com o risco de desenvolvimento de câncer já era conhecida, mas sua inclusão na lista negra da OMS serve de alerta para os brasileiros, que, segundo a nutricionista Sueli Couto, da Unidade Técnica de Alimentação, Nutrição e Câncer do INCA, têm aumentado o consumo de embutidos. mesmo aqueles produzidos a partir de carnes brancas como peito de peru defumado, blanquet, e outros, são prejudiciais à saúde. Esses produtos passam pelo mesmo processo industrial que os feitos para a carne vermelha. São adicionadas substâncias para realçar o sabor e dar mais tempo nas prateleiras. (INCA, 2015).

\section{CONCLUSÃO}

De acordo com a revisão proposta pelo presente trabalho, identificou-se que os compostos químicos presentes nas carnes embutidas (presunto, salsicha, linguiça, bacon, salame, mortadela, peito de peru e blanquet de peru) representam um grave problema de saúde pública nos tempos atuais, correlacionando-se com aspectos etiológicos, epidemiológicos e terapêuticos bastante complexos e fazendo correlação direta ao desenvolvimento do câncer colorretal. Uma vez que os compostos chamados nitritos e nitratos causam mudanças celulares no trato digestório e ingerindo esses alimentos com frequência aumentam a chances de desenvolver uma patologia associada.

Evidências científicas têm sido avaliadas e sumarizadas em recomendações pelo INCA e pela Organização Mundial da Saúde as quais mostram que o consumo desses produtos deve ser mínimo para que se possa reduzir o risco de desenvolvimento do câncer colorretal, principalmente, para as pessoas pertencentes aos grupos de risco e com predisposição genética. Lembrando, ainda, que os mesmos processos industriais aplicados 
aos embutidos são, hoje, utilizados na preparação de todas as carnes processadas, sejam elas provenientes de carnes vermelhas ou brancas.

\section{REFERÊNCIAS BIBLIOGRÁFICAS}

BOSTON.2015.Disponívelem:https://www.hsph.harvard.edu/nutritionsource/2015/rI/o3/ report-says-eating-processed-meat-is -the-findings/. Acessado em I6/10/2021

BRASIL. Ministério da Saúde. Instituto Nacional de Câncer. Falando sobre câncer do intestino. Rio de Janeiro: INCA, 2003.

BARBOSA, J. O que são embutidos? Origem, evolução e legislação. Disponível em: Rio de Janeiro, 2018. Disponível em: https://foodsafetybrazil.org/o-que-sao-embutidos-origemevolucao-e-legislacao/. Acessado em I2/10/2021.

FRANCO, B.; LANDGRAF, M. Microbiologia dos alimentos. Editora Atheneu. São Paulo.1996.

GARÓFOLO, A.; AVESANI, C.; CAMARGO, K.; BARROS, et al. Dieta e câncer: um enfoque epidemiológico. Revista de Nutrição / Pontifícia Universidade Católica de Campinas, Campinas, 2005. Disponível em:https://www.scielo.br/j/rn/a/ ?lang=pt

HENDGES, D.; STOLL, R.; MORESCHI, C. A influência de hábitos e estilo de vida no surgimento de neoplasias malignas - Uma revisão de literatura. Revista Destaques Acadêmicos, vol. $5, \quad \mathrm{n} \times \quad 3, \quad 2013 \quad$ - CCBS/UNIVATES. Disponível em:http://www.univates.br/article/view/288

INCA - Instituto Nacional do Câncer, Ministério da Saúde. OMS classifica carnes processadas como cancerígenas. Rio de Janeiro,2018.Disponívelem:https://www.inca.gov.br/omo-cancerigenas. Acesso em I2/10/2021.

INCA - Instituto Nacional de Câncer, Ministério da Saúde. Estimativa 2020 - Síntese dos Resultados e Comentários. Publicado em 24/02/2020. Disponível em:https://www.inca.gov.br/estimativa/sintese-de-resultados-e-comentarios.

INCA - Instituto Nacional de Câncer, Ministério da Saúde. Câncer do Intestino - Versão para Profissionais de Saúde. Publicado em 23/08/2021. Disponível em:https://www.inca.gov.br/tipos-de-cancer/cancer-de-intestino/profissional-de-saude.

Acesso em I2/10/2021.

MENEZES, C.; FERREIRA, D.; FARO, F.; BOMFIM, M.; TRINDADE, L. Câncer Colorretal na população brasileira: taxa de mortalidade no período de 2005-2015. Revista Brasileira em Promoção da Saúde, Universidade de Fortaleza. vol. 29, núm. 2, abril-junho, 2016, pp. 172-179. 
RIBEIRO, E. P., SERAVAlli, E. A. G. Química de alimentos. São Paulo: Edgard Blücler, Instituto Mauá de Tecnologia, 2004.

SILVA, F.; SOUSA, A.; SIQUEIRA, S. Câncer Colorretal: Promoção, Prevenção e Rastreamento. Revista Científica FacMais, Volume XIII, Número 2. Junho. Ano 2018/1을 Semestre. ISSN 2238-8427. Disponível em:http://revistacientifica.facmais.com.br/wpcontent/uploads/2018/10/6.C\% $\mathrm{C}_{3} \% 82 \mathrm{NCERCOLORRETALPROMO} \% \mathrm{C}_{3} \% 87 \% \mathrm{C}_{3} \% 83 \mathrm{O}$ -PREVEN\%C3\%87\%C3\%83O-E-RASTREAMENTO.pdf.

SPRINGHOUSE, C. Fisiopatologia: série incrivelmente facil!. Rio de Janeiro: Guanabara Koogan, 2004. In: HENDGES, D.; STOLL, R.; MORESCHI, C. A influência de hábitos e estilo de vida no surgimento de neoplasias malignas - Uma revisão de literatura. Revista Destaques Acadêmicos, vol. 5, no 3, p.122-124.CCBS/UNIVATES. 2013.

UNDERFERTH, D. Processed meat and cancer: What you need to know. MD Cancer Center. University of Texas. Houston. 2016. Disponível em: https://www.mdanderson.org/publications/focusedonhealth/eatlessprocessedmeat.hiris9 o624.html\#: :text=Processed\%20meat\%20and\%2ocancer\%3A\%2oWhat\%2oyou\%2oneed\%2 oto,dogs.\%2oEating\%2oprocessed\%20 meats\%2oincreases\%20your\%20cancer\%2orisk.

Acessado em I7 de out. de 202I.

WHO. World Health Organization. Q\&A on the carcinogenicity of the consumption of red meat and processed meat. International Agency for Reseach on Cancer, Lyon. 2015

WHO. World Health Organization. Links between processed meat and colorectal cancer. Publicado em: 29/10/2015. Disponível em: https://www.who.int/news/item/29-10-2015links-between-processed-meat-and-colorectal-cancer. Acesso em: 20/II/202I.

WHO. World Health Organization. WHO report says eating processed meat is carcinogenic: Understanding the findings. Harvard T.H.Chan. School of Public Health.HarvardSchool.https://www.hsph.harvard.edu/nutritionsource/2015/II/o3/reportsays-eating-processed-meat-is-carcinogenic-understanding-the-findings/ 